\title{
B.Ye. Rytsar
}

\section{A Simple Numeric Set-Theoretical Method of the Logic Differential Calculus}

\begin{abstract}
|Предложен новый метод логического дифференциального исчисления на основании числового теоретико-множественного подхода к определению разных типов логических производных произвольного порядка от булевой функции. Алгоритм имеет относительно меньшую вычислительную сложность при меньшем количестве операций, необходимых для решения задачи, что подтверждено примерами определения разных логических производных произвольных порядков от функций, заимствованных авторами из известных публикаций в порядке сравнения с предложенным методом.

A new method of the logic differential calculus, based on the numeric set-theoretical approach for the definition of the different logic derivatives types of the arbitrary order for Boolean function is proposed. The given algorithm has relatively less computational complexity due to the lower amount of the operations required for solving the given problem. This is proved in the given examples borrowed from the well-known publications, in order to compare them with the proposed numeric set-theoretic method to obtain the different types of logic derivatives of arbitrary order.

Запропоновано новий метод логічного диференційного числення на основі числового теоретико-множинного підходу до визначення різних типів логічних похідних довільного порядку від бульової функції. Алгоритм має відносно меншу обчислювальну складність за меншої кількості операцій, необхідних для розв'язання задачі, що підтверджено прикладами визначення запропонованим методом різних логічних похідних довільних порядків від функцій, запозичених авторами з відомих публікацій з метою порівняння з пропонованим методом.
\end{abstract}

Introduction. The modern methods of the logic design of digital devices and systems more often include the elements of the logic differential calculus [1-21]. This is related to qualitatively new possibilities of informational technologies and, consequently, to solving new problems of design and analysis of dynamic systems. In the survey $[5,7]$ the main directions, modern achievements and tendencies of the research in various branches of science and engineering is represented and fully analysed. Among them there are recearch on sensitivity of the combination network to changes of separate variables or their groups of the logic function that describes [9], testing the logic schema aimed at determining its faultiness at its arbitrary point [10-12], functional decomposition [1,11,13], minimization in the class of Reed-Muller's forms of different polarity [14], identification of symmetry $[15,16]$, and different optimization of problems of logic synthesis of digital devices and systems $[12,17,18]$, etc.

The main obstacle to wide application of the logic derivatives in different aspects of research is the complexity of the practical realization of their determination (calculation) in case of high orders and large number of variables [9]. Traditional symbolic transformations or visual methods based on Karnaugh maps [2-4] and patterns of Boolean functions [20] do not satisfy the requirements of the modern practice. Determination of the logic derivatives from the axioms and theorems of Boolean algebra and simplification of obtained analytical expressions is quite difficult and tedious. The application of matrix device based in particular on FFT-like algorithms enabled us to automate the process of the logic differential calculus $[3,5,8,21]$. However, as mentioned in [5, 7], the realization of the matrix transformations in the case of many variables is difficult, and in addition, cumbersome, since the calculation of Kronecker product, which is used for this, even in case of a simple derivative with respect to one variable for a function of $n$ variables the dimension of the matrix must be $2^{n} \times 2^{n}$. These shortcomings become particularly noticeable when calculating the logic derivatives of high orders.

We represent a new method of the logic differential calculus based on the numeric set-theoretical approach for definition of different logic derivatives types of the arbitrary order for Boolean function. The proposed method is compared with the known analytical, visual and matrix methods, relatively simpler as to its practical realization manually as well as with the assistance of computer without any previous transformations of Boolean function given in set-theoretical format. The presented algorithm has relatively less computational complexity due to lower amount of the operations required. 
The paper is organized as follows. Section 1 introduces the main theoretical part of the well-known definition of the different types of logic derivatives. Section 2 presents the proposed numeric settheoretical (ST) method. This section discusses the theoretical basis of the method (p. 2.1), obtaining the simple ST-derivative (p. 2.2), obtaining the multiple ST-derivative (p. 2.3), obtaining the vectorial STderivative of the $k$-th order (p. 2.4). This section also describes the algorithm of the method and examples to illustrate the definition of the different logic derivatives types of the various orders.

\section{The basic theoretical part}

In the basis of calculation of logic derivative there is the notion of change of a Boolean function $f\left(x_{1}, x_{2}, \ldots, x_{n}\right)$ with respect to one of its independent variables $x_{i} \in\{0,1\}$ and this change $(0 \rightarrow 1$ and $1 \rightarrow 0$ ) is equal to log. 1 . If such a modification does not change the value of the function $f \in\{0,1\}$, the logic derivative is equal to zero, i.e. $\partial f / \partial x_{i}=0$, otherwise $\partial f / \partial x_{i}=1$.

Let us consider the well-known definition of the main types of logic derivatives $[1-5,7,12,19]$.

The logic derivative for a function $f\left(x_{1}, x_{2}, \ldots, x_{n}\right)$ with respect to a variable $x_{i}$, the so-called simple derivation of the 1-st order $\partial f / \partial x_{i}$, one can determine by the operation of mod-2-sum:

$$
\frac{\partial f}{\partial x_{i}}=f\left(x_{1}, \ldots, x_{i}, \ldots, x_{n}\right) \oplus f\left(x_{1}, \ldots, \bar{x}_{i}, \ldots, x_{n}\right),
$$

where $f\left(x_{1}, \ldots, x_{i}, \ldots, x_{n}\right)$ is a given function and $f\left(x_{1}, \ldots, \bar{x}_{i}, \ldots, x_{n}\right)$ is a function obtained by replacement of all $x_{i}$ for $\bar{x}_{i}$ and all $\bar{x}_{i}$ for $x_{i}$. For example, let $f\left(x_{1}, x_{2}, x_{3}\right)$ be a given function. Then $f\left(\bar{x}_{1}, x_{2}, x_{3}\right)$ is the function $f\left(x_{1}, x_{2}, x_{3}\right)$ mapping 3-tuple values $\left\langle\sigma_{1}, \sigma_{2}, \sigma_{3}\right\rangle$ of their variables $x_{1}, x_{2}, x_{3}$ into 3-tuple values $\left\langle\bar{\sigma}_{1}, \sigma_{2}, \sigma_{3}\right\rangle, \sigma_{i} \in\{0,1\}$. For example, the logic derivative (1) for $f\left(x_{1}, x_{2}, x_{3}\right)=x_{1} x_{2} \vee \bar{x}_{1} x_{3}$ with respect to $x_{1}$ is obtained in the following way:

$$
\begin{aligned}
\frac{\partial f}{\partial x_{1}} & =f\left(x_{1}, x_{2}, x_{3}\right) \oplus f\left(\bar{x}_{1}, x_{2}, x_{3}\right)=\left(x_{1} x_{2} \vee \bar{x}_{1} x_{3}\right) \oplus\left(\bar{x}_{1} x_{2} \vee x_{1} x_{3}\right)= \\
& =\bar{x}_{1} x_{2} \bar{x}_{3} \vee x_{1} \bar{x}_{2} x_{3} \vee x_{1} x_{2} \bar{x}_{3} \vee \bar{x}_{1} \bar{x}_{2} x_{3}=\bar{x}_{2} x_{3} \vee x_{2} \bar{x}_{3}=x_{2} \oplus x_{3} .
\end{aligned}
$$

A similar result can be obtained if we rewrite (1) by taking into account equality $x_{i}=1$ and $\bar{x}_{i}=0$ for a function $f\left(x_{1}, \ldots, x_{i}, \ldots, x_{n}\right): \quad \frac{\partial f}{\partial x_{i}}=f\left(x_{1}, \ldots, x_{i-1}, 1, x_{i+1}, \ldots, x_{n}\right) \oplus f\left(x_{1}, \ldots, x_{i-1}, 0, x_{i+1}, \ldots, x_{n}\right)$.

We illustrate this in the example discussed above using the equality $x_{1}=1$ and $\bar{x}_{1}=0$ :

$$
\frac{\partial}{\partial x_{1}}\left(x_{1} x_{2} \vee \bar{x}_{1} x_{3}\right)=\left(1 x_{2} \vee 0 x_{3}\right) \oplus\left(0 x_{2} \vee 1 x_{3}\right)=x_{2} \oplus x_{3} \text {. }
$$

The multiple logic difference ( $k$-fold derivative) for a function $f$ with respect to some subset of $k$ variables $x_{i_{1}}, x_{i_{2}}, \ldots, x_{i_{k}}$ is given by the expression

$$
\frac{\partial^{k} f}{\partial x_{i_{1}} \partial x_{i_{2}} \cdots \partial x_{i_{k-1}} \partial x_{i_{k}}}=\frac{\partial}{\partial x_{i_{k}}}\left(\frac{\partial^{k-1} f}{\partial x_{i_{1}} \partial x_{i_{2}} \cdots \partial x_{i_{k-1}}}\right) .
$$

The multiple derivative of $k$ orders (2) is calculated by (1) of $k$ times fixing in turn variables $x_{i_{1}}, x_{i_{2}}, \ldots, x_{i_{k}}$ in arbitrary order:

$$
\frac{\partial^{k} f}{\partial x_{i_{1}} \partial x_{i_{2}} \cdots \partial x_{i_{k}}}=\frac{\partial}{\partial x_{i_{1}}}\left(\frac{\partial}{\partial x_{i_{2}}}\left(\cdots\left(\frac{\partial}{\partial x_{i_{k}}}\right) \cdots\right)\right) \text {. }
$$


For example, $\frac{\partial^{2}}{\partial x_{1} \partial x_{2}}\left(\bar{x}_{1} \bar{x}_{2} \bar{x}_{3}\right)=\frac{\partial}{\partial x_{2}}\left(\frac{\partial}{\partial x_{1}}\left(\bar{x}_{1} \bar{x}_{2} \bar{x}_{3}\right)\right)=\frac{\partial}{\partial x_{2}}\left(\bar{x}_{2} \bar{x}_{3}\right)=\bar{x}_{3}$.

The vectorial derivative of the $k$-th order $\frac{\partial^{k} f}{\partial\left(x_{i_{1}}, x_{i_{2}}, \ldots, x_{i_{k}}\right)}$ for a function $f\left(x_{1}, x_{2}, \ldots, x_{n}\right)$ with respect to some $k$ variables $x_{\lambda_{1}}, x_{\lambda_{2}}, \ldots, x_{\lambda_{k}}$ determines the conditions in which the function $f$ changes its value with simultaneous change of values of these $k$ variables. The vectorial derivative of the $k$-th order for the function $f$ with respect to variables $x_{\lambda_{1}}, x_{\lambda_{2}}, \ldots, x_{\lambda_{k}}$ is equal to the mod-2-sum of all variables of the 1-th, 2 -nd, ..., $k$-th orders of multiple derivatives with respect to the fixed variables $x_{\lambda_{1}}, x_{\lambda_{2}}, \ldots, x_{\lambda_{k}}$ :

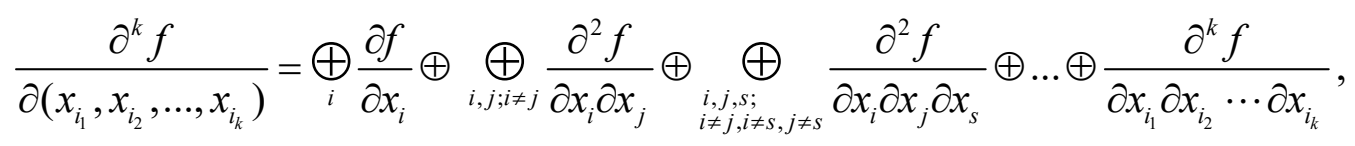

where $i, j, s, \ldots=i_{1}, i_{2}, \ldots, i_{k}$.

Thus, if there is a need to find out in which conditions the value, for example, of a function $f\left(x_{1}, x_{2}, x_{3}\right)$ will change in the case of simultaneous change of values of all its variables, then it is necessary to calculate the derivative of the 3-rd order of the type:

$$
\frac{\partial^{3} f}{\partial\left(x_{1}, x_{2}, x_{3}\right)}=\frac{\partial f}{\partial x_{1}} \oplus \frac{\partial f}{\partial x_{2}} \oplus \frac{\partial f}{\partial x_{3}} \oplus \frac{\partial^{2} f}{\partial x_{1} \partial x_{2}} \oplus \frac{\partial^{2} f}{\partial x_{1} \partial x_{3}} \oplus \frac{\partial^{2} f}{\partial x_{2} \partial x_{3}} \oplus \frac{\partial^{3} f}{\partial x_{1} \partial x_{2} \partial x_{3}},
$$

where after calculations of all derivatives of the 1-st order $\frac{\partial f}{\partial x_{1}}, \frac{\partial f}{\partial x_{2}}, \frac{\partial f}{\partial x_{3}}$ and multiple derivatives of the 2-nd order $\frac{\partial^{2} f}{\partial x_{1} \partial x_{2}}=\frac{\partial}{\partial x_{2}}\left(\frac{\partial f}{\partial x_{1}}\right), \frac{\partial^{2} f}{\partial x_{1} \partial x_{3}}=\frac{\partial}{\partial x_{3}}\left(\frac{\partial f}{\partial x_{1}}\right), \frac{\partial^{2} f}{\partial x_{2} \partial x_{3}}=\frac{\partial}{\partial x_{3}}\left(\frac{\partial f}{\partial x_{2}}\right)$ the required derivative of the 3-rd order one can calculate through multiple derivative, for example, $\frac{\partial^{2} f}{\partial x_{1} \partial x_{2}}$, thus: $\frac{\partial^{3} f}{\partial x_{1} \partial x_{2} \partial x_{3}}=\frac{\partial}{\partial x_{3}}\left(\frac{\partial^{2} f}{\partial x_{1} \partial x_{2}}\right)$.

From the considered above the properties of logic derivatives that are quite obvious to prove follow out. In particular, since by definition (1) the values of logic derivatives of a function $f$ of $n$ variables with respect to $x_{i}$ and $\bar{x}_{i}$ are the same, it is obvious that the resulting function is independent of the variable $x_{i}$ and is a function of $(n-1)$ variables. On the other hand, if $\partial f / \partial x_{i}=0$ the function $f$ is independent of the variable $x_{i}$, i.e., $x_{i}$ is an inessential variable. In contrast, if the same function $f$ does not depend on a variable $x_{i}$, then $\partial f / \partial x_{i}=0$. Apparently, $\partial f / \partial x_{i}=1$ if $f\left(x_{1}, \ldots, \bar{x}_{i}, \ldots, x_{n}\right)=\bar{f}\left(x_{1}, \ldots, x_{i}, \ldots, x_{n}\right)$ and, therefore, $x_{i}$ is an essential variable of the function $f$. In contrast to the classical concept of a derivative, the logic derivative does not distinguish the direction of change of the function $0 \rightarrow 1$ or $1 \rightarrow 0$, i.e. $\frac{\partial f}{\partial x_{i}}=\frac{\partial \bar{f}}{\partial x_{i}}$. In addition, the order of differentiation (2) with respect to different variables does not affect the result: $\frac{\partial^{2} f}{\partial x_{i} \partial x_{j}}=\frac{\partial^{2} f}{\partial x_{j} \partial x_{i}}$. The logic derivative of the 2-nd order with respect to the same variable $\partial^{2} f / \partial x_{i} \partial x_{i}=0$.

It should be mentioned that the analytic definition of the logic derivatives of $k$ orders for different types even for functions of several variables, as it is not difficult to find out from the mentioned above, requires considerable effort and time. Moreover, the complexity of such calculation increases rapidly with increasing of $n$. 


\section{Numeric set-theoretical method}

\subsection{Theoretical basis of the method}

In general, any logic function $f$ of $n$ variables, i.e., $f: X \rightarrow\{0,1\}, X=\left\{x_{1}, x_{2}, \ldots, x_{n}\right\}, x_{i} \in\{0,1\}$, can be given in the set-theoretical form (STF) of some set $Y^{1}$ of numeric (binary or decimal) conjuncterms (conjunctive terms) $\theta_{1}^{r_{1}}, \theta_{2}^{r_{2}}, \ldots, \theta_{p}^{r_{p}}$ of arbitrary ranks $r_{1}, r_{2}, \ldots, r_{p} \in\{1,2, \ldots, n\}, 1<p<2^{n}$, i.e. in the so-called set-theoretical form (STF) $Y^{1}=\left\{\theta_{1}^{r_{1}}, \theta_{2}^{r_{2}}, \ldots, \theta_{p}^{r_{p}}\right\}^{1}$, where $i$-th conjuncterm of the $r_{i}$-rank $\theta_{i}^{r_{i}}=\left(\sigma_{1} \sigma_{2} \cdots \sigma_{n}\right)_{i}$, $\sigma_{j} \in\{0,1,-\}$; the superscript $Y^{1}=\{\ldots\}^{1}$ indicates that the analytical equivalent of STF $Y^{1}$ is a sum-ofproduct (SOP) form of the function $f[23,24]$.

A particular case of STF $Y^{1}$ is the canonical STF $Y^{1}=\left\{m_{1}, m_{2}, \ldots, m_{p}\right\}^{1}$ of the function $f\left(x_{1}, x_{2}, \ldots, x_{n}\right)$, where $m_{1}, m_{2}, \ldots, m_{p}\left(m_{i}=\left(\sigma_{1} \sigma_{2} \cdots \sigma_{n}\right)_{i}, \sigma_{j} \in\{0,1\}\right)$, are binary minterms (i.e. conjuncterms of $n$-rank), whose values correspond to the values of the $n$-tuple $\left\langle\sigma_{1}, \sigma_{2}, \ldots, \sigma_{n}\right\rangle$, in which the value function $f=1$. For example, when a function $f\left(x_{1}, x_{2}, x_{3}\right)$ is given by a canonical STF $Y^{1}=\{(000),(010),(110)\}^{1}$, in this case $f=1$ for the 3 -tuples $\langle 0,0,0\rangle,\langle 0,1,0\rangle,\langle 1,1,0\rangle$ and for the rest of the 3-tuples $\langle 0,0,1\rangle,\langle 0,1,1\rangle$, $\langle 1,0,0\rangle,\langle 1,0,1\rangle,\langle 1,1,1\rangle$ the value $f=0$.

Let us consider the function given by a set of conjuncterms of different ranks, such as $f\left(x_{1}, x_{2}, x_{3}\right)$ having STF $Y^{1}=\{(011),(-0-)\}^{1}$, where (011) are binary minterms (conjuncterms of 3-rank), and $(-0-)$ are ternary conjuncterms of 1 -rank formed by four adjacent minterms $(000),(001),(100),(101)$, i.e. $(-0-)=\{(000),(001),(100),(101)\}$. In this case $f=1$ for the $n$-tuples $\langle 0,0,0\rangle,\langle 0,0,1\rangle,\langle 0,1,1\rangle,\langle 1,0,0\rangle$, $\langle 1,0,1\rangle$ and $f=0$ for the $n$-tuples $\langle 0,1,0\rangle,\langle 1,1,0\rangle,\langle 1,1,1\rangle$. Here, the dash (-) in the $i$-th position of ternary conjuncterms symbolizes the missing variable in its analytic expression $x_{i}$, i.e. $(-0-) \equiv \bar{x}_{2}$, where $x_{1}$ and $x_{3}$ are absent. Thus, the analytical equivalent of the STF $Y^{1}=\{(011),(-0-)\}^{1}$ is the SOP $f=\bar{x}_{1} x_{2} x_{3} \vee \bar{x}_{2}$.

The content of the proposed numeric set-theoretical method of logic differential calculus is based on the fact that in the simplest case, the logic derivative of the 1-st order with respect to the variable $x_{i}$ (1) for arbitrary binary minterm $\left(\sigma_{1} \cdots \sigma_{i} \cdots \sigma_{n}\right)$ or $\left(\sigma_{1} \cdots \bar{\sigma}_{i} \cdots \sigma_{n}\right), \sigma_{i} \in\{0,1\}$, of the function $f\left(x_{1}, \ldots, x_{i}, \ldots, x_{n}\right)$ can be considered as conjuncterm of the $(n-1)$-rank $\left(\sigma_{1} \cdots-_{i} \cdots \sigma_{n}\right)$ in which variable $x_{i}$ is absent:

$$
\frac{\partial}{\partial x_{i}}\left(\sigma_{1} \cdots \sigma_{i} \cdots \sigma_{n}\right)=\frac{\partial}{\partial x_{i}}\left(\sigma_{1} \cdots \bar{\sigma}_{i} \cdots \sigma_{n}\right)=\left(\sigma_{1} \cdots \sigma_{i} \cdots \sigma_{n}\right) \oplus\left(\sigma_{1} \cdots \bar{\sigma}_{i} \cdots \sigma_{n}\right)=\left(\sigma_{1} \cdots-{ }_{i} \cdots \sigma_{n}\right) \text {. }
$$

For example, due to logic differentiation of a minterm $\bar{x}_{1} \bar{x}_{2} \bar{x}_{3}$ or $x_{1} \bar{x}_{2} \bar{x}_{3}$ with respect to $x_{1}$ of a function $f\left(x_{1}, x_{2}, x_{3}\right)$ we have $\partial\left(\bar{x}_{1} \bar{x}_{2} \bar{x}_{3}\right) / \partial x_{1}=\partial\left(x_{1} \bar{x}_{2} \bar{x}_{3}\right) / \partial x_{1}=\bar{x}_{1} \bar{x}_{2} \bar{x}_{3} \oplus x_{1} \bar{x}_{2} \bar{x}_{3}=\left(\bar{x}_{1} \oplus x_{1}\right) \bar{x}_{2} \bar{x}_{3}=\bar{x}_{2} \bar{x}_{3}$, and in the numeric set-theoretical form for (000) or (100) have $\partial(000) / \partial x_{1}=\partial(100) / \partial x_{1}=(-00)$.

On this basis, one could argue that the logic derivative of a conjuncterm of the $(n-1)$-rank with respect to the missing variable is equal to zero, and in the numeric set-theoretical format is the empty set $(\varnothing)$. For example, a logic derivative of a conjuncterm of the $(n-1)$-rank $\bar{x}_{2} \bar{x}_{3}$ of a function $f\left(x_{1}, x_{2}, x_{3}\right)$ with respect to $x_{1}$ will be $\partial\left(\bar{x}_{2} \bar{x}_{3}\right) / \partial x_{1}=\bar{x}_{2} \bar{x}_{3} \oplus \bar{x}_{2} \bar{x}_{3}=0$, and in the set-theoretical form $\partial(-00) / \partial x_{1}=\varnothing$.

Respectively, if a function $f$ is given in set-theoretical format by $p$ binary minterms, i.e. a canonical STF $Y^{1}$, due to logic differentiation with respect to the variable $x_{1}$ in set-theoretical format we obtain a set of $p$ ternary conjuncterms of the $(n-1)$-rank. 
Set-theoretical procedure of obtaining of logic derivatives of different types of the $k$-th order $(k=1,2, \ldots, n)$ (see Section 1$)$ for a function $f\left(x_{1}, x_{2}, \ldots, x_{n}\right)$ that is given by a canonical STF $Y^{1}$ will be called set-theoretical logic differentiation (ST-differentiation). The result of ST-differentiation of a logic derivative of the $k$-th order will be called the multiple ST-derivative of the $k$-th order. If $k=1$, i.e., a simple ST-derivative, which is denoted as $\partial Y^{\oplus} / \partial x_{i}$, where the superscript $\oplus$ represents the logic operation by mod-2-sum, as the established set contains conjuncterms considered in the so-called polynomial set-theoretic form $(P S T F) Y^{\oplus}[23,24]$. For example, PSTF $Y^{\oplus}=\{(-00),(-10)\}^{\oplus}=(--0)$ corresponds to $f\left(x_{1}, x_{2}, x_{3}\right)=\bar{x}_{2} \bar{x}_{3} \oplus x_{2} \bar{x}_{3}=\bar{x}_{3}$.

The procedure for ST-differentiation is convenient to implement by "overlaying" $n$-position mask of literals on binary minterms of a canonical STF $Y^{1}$ of a function $f$ [22]. In the case of ST-differentiation with respect to one variable $x_{i}$ (the operator $\stackrel{\partial / \partial x_{i}}{\Rightarrow}$ ), such a mask will be designated as a conjunction of literals $\left\{l_{1} \cdots l_{i-1}-l_{i+1} \cdots l_{n}\right\}, l_{j} \in\{0,1\}$, where in the $i$-th position literal is replaced by the character dash $(-)$. Thus, the ST-differentiation procedure, in particular, for the binary minterm $\left(\sigma_{1} \cdots \sigma_{i} \cdots \sigma_{n}\right)$ of a function $f\left(x_{1}, \ldots, x_{i}, \ldots, x_{n}\right)$ with respect to the variable $x_{i}$ has the following form

$$
Y^{1}=\left\{\left(\sigma_{1} \cdots \sigma_{i} \cdots \sigma_{n}\right)\right\}^{1 / \partial \partial x_{i}} \Rightarrow\left\{l_{1} \cdots l_{i-1}-l_{i+1} \cdots l_{n}\right\}=\left\{\left(\sigma_{1} \cdots-{ }_{i} \cdots \sigma_{n}\right)\right\}^{\oplus},
$$

where the desired simple ST-derivative $\partial Y^{\oplus} / \partial x_{i}=\left(\sigma_{1} \cdots-_{i} \cdots \sigma_{n}\right)$ corresponds to the derivative of the 1-st order $\partial f / \partial x_{i}=f^{\prime}\left(x_{1}, \ldots, x_{i-1}, x_{i+i}, \ldots, x_{n}\right)$.

Respectively, when determining the ST-derivative of the $k$-th order, the mask of literals will have the dash (-) in $k$ positions that belong to $k$ variables, for which the derivative is sought.

\subsection{Obtaining of the simple ST-derivative}

As described in Section 2.1 in order to obtain a simple ST-derivative for a function $f\left(x_{1}, \ldots, x_{i}, \ldots, x_{n}\right)$ with respect to the variable $x_{i}$ (operator $\stackrel{\partial / \partial x_{i}}{\Rightarrow}$ ), it is sufficient to put on binary minterms $m_{1}, m_{2}, \ldots, m_{p}$ of canonical STF $Y^{1}=\left\{m_{1}, m_{2}, \ldots, m_{p}\right\}^{1}$ the mask $\left\{l_{1} \cdots l_{i-1}-l_{i+1} \cdots l_{n}\right\}$. As a result, in the polynomial format there is produced a set of ternary conjuncterms of the $(n-1)$-rank $\theta_{1}^{n-1}, \theta_{2}^{n-1}, \ldots, \theta_{p}^{n-1}$ with the dash (-) in the $i$-th position, i.e. PSTF $Y^{\oplus}=\left\{\theta_{1}^{n-1}, \theta_{2}^{n-1}, \ldots, \theta_{p}^{n-1}\right\}^{\oplus}$. The resulting PSTF $Y^{\oplus}$ can be simplified to a form $\left\{\theta_{1}^{n-1}, \theta_{2}^{n-1}, \ldots, \theta_{p^{*}}^{n-1}\right\}, p^{*} \leq p$, by eliminating identical pairs of elements according to the rule

$$
\left\{\theta_{i}^{n-1}, \theta_{j}^{n-1}\right\}^{\oplus}=\left\{\begin{array}{ll}
\varnothing, & \text { if } \theta_{i}^{n-1}=\theta_{j}^{n-1} \\
\theta_{i}^{n-1}, \theta_{j}^{n-1}, & \text { if } \theta_{i}^{n-1} \neq \theta_{j}^{n-1}
\end{array} .\right.
$$

The procedure for obtaining the simple ST-derivative $\partial Y^{\oplus} / \partial x_{i}$ for the function $f$, that is given by a canonical STF $Y^{1}$, is the following:

$$
\begin{gathered}
Y^{1}=\left\{m_{1}, m_{2}, \ldots, m_{p}\right\}^{1 / \partial \partial x_{i}} \stackrel{\Rightarrow}{\Rightarrow}\left\{l_{1} \cdots l_{i-1}-l_{i+1} \cdots l_{n}\right\}=\left\{\theta_{1}^{n-1}, \theta_{2}^{n-1}, \ldots, \theta_{p}^{n-1}\right\}^{\oplus} \Rightarrow\left\{\theta_{1}^{n-1}, \theta_{2}^{n-1}, \ldots, \theta_{p^{*}}^{n-1}\right\}^{\oplus} \Rightarrow \\
\Rightarrow\left\{\theta_{1}^{r_{1}}, \theta_{2}^{r_{2}}, \ldots, \theta_{s}^{r_{s}}\right\},
\end{gathered}
$$

where $\left\{\theta_{1}^{r_{1}}, \theta_{2}^{r_{2}}, \ldots, \theta_{s}^{r_{s}}\right\}$ is the set of conjuncterms of the different $\left(r_{1}, r_{2}, \ldots, r_{s}\right)$ ranks formed by minimization procedure, i.e. that is the desired simple ST-derivative $\partial Y^{\oplus} / \partial x_{i}$ for the function $f$. 
It should be noted that the superscript in the resulting set of (6) is dropped. This shows that the minimization procedure can be performed either in polynomial (PSTF $Y^{\oplus}$ ) or in disjunctive format (STF $Y^{1}$ ) as the elements of the set $\left\{\theta_{1}^{n-1}, \theta_{2}^{n-1}, \ldots, \theta_{p^{*}}^{n-1}\right\}$ are mutually orthogonal conjuncterms.

In the examples below the minimization procedure will be implemented in the STF $Y^{1}$.

Example 1. Calculate a simple logic derivative for the function $f=\bar{x}_{1} \bar{x}_{2} \bar{x}_{3} \vee \bar{x}_{1} x_{2} \bar{x}_{3} \vee x_{1} x_{2} \bar{x}_{3}$ with respect to variable $x_{2}$ by numeric set-theoretical method (this fis borrowed from [20], p. 128).

Solution. The given function $f$ has canonical STF $Y^{1}=\{(000),(010),(110)\}^{1}$. Applying the mask $\left\{l_{1}-l_{3}\right\}$ on its minterms we get: $Y^{1}=\{(000),(010),(110)\}^{1 / \partial x_{2}} \stackrel{\Rightarrow}{\Rightarrow}\left\{l_{1}-l_{3}\right\}=\{(0-0),(0-0),(1-0)\}^{\oplus} \Rightarrow\{(1-0)\}$.

Answer. The simple ST-derivative $\partial Y^{\oplus} / \partial x_{2}=(1-0)$ corresponds to the derivative $\partial f / \partial x_{2}=x_{1} \bar{x}_{3}$ [20].

If the function $f$ is given in the SOP or STF $Y^{1}$, its conjuncterms should be mutually orthogonalized [2123] or turned into the canonical form. We illustrate this in the example described in Section 2, the definition of simple ST-derivative for $f\left(x_{1}, x_{2}, x_{3}\right)=x_{1} x_{2} \vee \bar{x}_{1} x_{3}$ with respect to the variable $x_{1}$. Since this function has orthogonal conjuncterms having written its STF $Y^{1}=\{(11-),(0-1)\}^{1}$ according to (6), we obtain

$$
Y^{1}=\{(11-),(0-1)\}^{1 / \partial \partial x_{1}} \Rightarrow\left\{-l_{2} l_{3}\right\}=\{(-1-),(--1)\}^{\oplus},
$$

that corresponds to the analytical expression $\partial f / \partial x_{1}=x_{2} \oplus x_{3}$.

The same result will be obtained for the canonical STF $Y^{1}$ of this function $f$ :

$$
Y^{1}=\{(001),(011),(110),(111)\}^{1 / \partial / x_{1}} \Rightarrow\left\{-l_{2} l_{3}\right\}=\{(-01),(-11),(-10),(-11)\}^{\oplus}=\{(--1),(-1-)\} .
$$

Example 2. Calculate all simple logic derivatives for the function $f=x_{1} x_{2} \oplus x_{3} x_{4}$ by numeric settheoretical method (this $f$ is borrowed from [25], p. 46).

Solution. The given function $f$ has PSTF $Y^{\oplus}=\{(11--),(--11)\}^{\oplus}$, on the basis of which the required derivatives are obtained in the following way:

$$
\begin{aligned}
& Y^{\oplus} \stackrel{\partial / \partial x_{1}}{\Rightarrow}\left\{-l_{2} l_{3} l_{4}\right\}=(-1--), \text { i.e. } \partial f / \partial x_{1}=x_{2} ; Y^{\oplus} \stackrel{\partial / \partial x_{2}}{\Rightarrow}\left\{l_{1}-l_{3} l_{4}\right\}=(1--), \text { i.e. } \partial f / \partial x_{2}=x_{1} ; \\
& Y^{\oplus} \stackrel{\partial / \partial x_{3}}{\Rightarrow}\left\{l_{1} l_{2}-l_{4}\right\}=(---1), \text { i.e. } \partial f / \partial x_{3}=x_{4} ; Y^{\oplus / \partial x_{4}} \stackrel{\Rightarrow}{\Rightarrow}\left\{l_{1} l_{2} l_{3}-\right\}=(--1-), \text { i.e. } \partial f / \partial x_{4}=x_{3} .
\end{aligned}
$$

Answer. The simple logic derivatives obtained by numeric set-theoretical method corresponds to [25].

\subsection{Obtaining of multiple ST-derivative}

In set-theoretic form the multiple logic derivative of the $k$-th order (2) for a function $f\left(x_{1}, x_{2}, \ldots, x_{n}\right)$ will be called the multiple ST-derivative of the $k$-th order. Based on the above consideration, the obtaining of the multiple ST-derivative can be interpreted as the procedure of conjuncterms of the $(n-k)$-rank $(k \leq n)$ formation in PSTF $Y^{\oplus}$. To confirm this we will consider the definition of the 2-nd order multiple derivative for the function $f\left(x_{1}, \ldots, x_{i}, \ldots, x_{j}, \ldots, x_{n}\right)$ with respect to the variables $x_{i}$ and $x_{j}$. In view of the formulas (1) and (2) we have:

$$
\frac{\partial^{2} f}{\partial x_{i} \partial x_{j}}=\frac{\partial}{\partial x_{j}}\left(\frac{\partial f}{\partial x_{i}}\right)=\frac{\partial}{\partial x_{j}}\left(f\left(x_{1}, \ldots, x_{i}, \ldots, x_{j}, \ldots, x_{n}\right) \oplus f\left(x_{1}, \ldots, \bar{x}_{i}, \ldots, x_{j}, \ldots, x_{n}\right)\right),
$$

and, applying the rule (4) and taking into account the expressions $x_{i} \oplus \bar{x}_{i}=1$ and $x_{j} \oplus \bar{x}_{j}=1$, we obtain

$$
\frac{\partial}{\partial x_{j}}\left(f\left(x_{1}, \ldots, x_{i}, \ldots, x_{j}, \ldots, x_{n}\right)\right) \oplus \frac{\partial}{\partial x_{j}}\left(f\left(x_{1}, \ldots, \bar{x}_{i}, \ldots, x_{j}, \ldots, x_{n}\right)\right)=
$$




$$
\begin{gathered}
=\left(f\left(x_{1}, \ldots, x_{i}, \ldots, x_{j}, \ldots, x_{n}\right) \oplus f\left(x_{1}, \ldots, x_{i}, \ldots, \bar{x}_{j}, \ldots, x_{n}\right)\right) \oplus\left(f\left(x_{1}, \ldots, \bar{x}_{i}, \ldots, x_{j}, \ldots, x_{n}\right) \oplus f\left(x_{1}, \ldots, \bar{x}_{i}, \ldots, \bar{x}_{j}, \ldots, x_{n}\right)\right)= \\
=f^{\prime}\left(x_{1}, \ldots, x_{i}, \ldots, x_{j-1}, x_{j+1}, \ldots, x_{n}\right) \oplus f^{\prime}\left(x_{1}, \ldots, \bar{x}_{i}, \ldots, x_{j-1}, x_{j+1}, \ldots, x_{n}\right)=f^{\prime \prime}\left(x_{1}, \ldots, x_{i-1}, x_{i+1}, \ldots, x_{j-1}, x_{j+1}, \ldots, x_{n}\right) .
\end{gathered}
$$

Thus, the 2-nd order multiple derivative with respect to the variables $x_{i}$ and $x_{j}$ is:

$$
\frac{\partial^{2}}{\partial x_{i} \partial x_{j}}\left(f\left(x_{1}, \ldots, x_{i}, \ldots, x_{j}, \ldots, x_{n}\right)\right)=f^{\prime \prime}\left(x_{1}, \ldots, x_{i-1}, x_{i+1}, \ldots, x_{j-1}, x_{j+1}, \ldots, x_{n}\right) \text {. }
$$

For example, the 2-nd order multiple ST-derivative (2) of the function $f\left(x_{1}, x_{2}, x_{3}\right)=\bar{x}_{1} \bar{x}_{2} \bar{x}_{3}$ (see the example of Section 1) is defined as: $\frac{\partial^{2}}{\partial x_{1} \partial x_{2}}\left(\bar{x}_{1} \bar{x}_{2} \bar{x}_{3}\right)=\frac{\partial}{\partial x_{2}}\left(\frac{\partial}{\partial x_{1}}\left(\bar{x}_{1} \bar{x}_{2} \bar{x}_{3}\right)\right)=\frac{\partial}{\partial x_{2}}\left(\bar{x}_{2} \bar{x}_{3}\right)=\bar{x}_{3} \equiv\{(--0)\}^{1}$.

Therefore, in order to obtain the 2-nd order multiple ST-derivative with respect to $x_{i}$ and $x_{j}$, i.e. $\partial^{2} Y^{\oplus} / \partial x_{i} \partial x_{j}$, it is necessary to apply a mask of literals with symbols (-) in $i$-th and $j$-th positions to binary minterms $m_{1}, m_{2}, \ldots, m_{p}$ of canonical STF $Y^{1}$ of function $f$, i.e. $\left\{l_{1} \cdots l_{i-1}-l_{i+1} \cdots l_{j-1}-l_{j+1} \cdots l_{n}\right\}$. Thus, this procedure (the operator $\stackrel{\partial^{2} / \partial x_{i} \partial x_{j}}{\Rightarrow}$ ) is similar to the procedure for obtaining of the simple ST-derivative (6):

$$
\left.Y^{1}=\left\{m_{1}, m_{2}, \ldots, m_{p}\right\}^{\hat{\partial}^{2} / \partial x_{i} \partial x_{2}} \Rightarrow l_{1} \cdots l_{i-1}-l_{i+1} \cdots l_{j-1}-l_{j+1} \cdots l_{n}\right\}=\left\{\theta_{1}^{n-2}, \theta_{2}^{n-2}, \ldots, \theta_{p}^{n-2}\right\}^{\oplus},
$$

where $\theta_{1}^{n-2}, \theta_{2}^{n-2}, \ldots, \theta_{p^{*}}^{n-2}$ are the ternary conjuncterms of the $(n-2)$-rank of the formed PSTF $Y^{\oplus}$; $\theta_{1}^{n-2}, \theta_{2}^{n-2}, \ldots, \theta_{p^{*}}^{n-2}$ are the ternary conjuncterms of $(n-2)$-rank obtained after removal procedure of the identical pairs; $\left\{\theta_{1}^{r_{1}}, \theta_{2}^{r_{2}}, \ldots, \theta_{s}^{r_{s}}\right\}$ is a set of conjuncterms of different $\left(r_{1}, r_{2}, \ldots, r_{s}\right)$ ranks formed by minimization procedure in polynomial (PSTF $Y^{\oplus}$ ) or disjunctive (STF $Y^{1}$ ) format, which is a multiple STderivative of the 2-nd order $\partial^{2} Y^{\oplus} / \partial x_{i} \partial x_{j}$ for the function $f$.

Respectively, in order to obtain a multiple ST-derivative of the $k$-th order $\partial^{k} Y^{\oplus} / \partial x_{i_{1}} \partial x_{i_{2}} \cdots \partial x_{i_{k}}$ for the function $f$, given by canonical STF $Y^{1}$, we need to overlay on its binary minterms the mask of literals which has $k$ the dash (-) for those positions that, according to the $n$-tuple $\left\langle\sigma_{1}, \sigma_{2}, \ldots, \sigma_{n}\right\rangle, \sigma_{i} \in\{0,1\}$, belong to the variables with respect to which the derivative is searched.

We should note that in contrast to known methods to obtain a multiple ST-derivative of the $k$-th order by the proposed set-theoretical method, we do not need to perform step-by-step identification of appropriate derivatives of the first order (2).

An algorithm of obtaining a multiple ST-derivative of the $k$-th order $(k \in\{1,2, \ldots, n\})$ for function $f\left(x_{1}, x_{2}, \ldots, x_{n}\right)$ by numeric set-theoretical method is realized in the following way:

- on binary minterms of canonical STF $Y^{1}$ of the given function $f$ overlay a mask of literals with symbols (-) in $k$ positions that belong to $k$ variables with respect to which the function $f$ is differentiated;

- in the formed the PSTF $Y^{\oplus}$ the pairs of identical ternary conjuncterms of the $(n-k)$-rank are removed;

- the minimization procedure is executed on simplified of the set PSTF $Y^{\oplus}$ (or STF $Y^{1}$ ) and as a result of which the multiple ST-derivative for given function $f$ is obtained.

The presented below examples illustrate the proposed numeric set-theoretical method of obtaining multiple logic derivatives of arbitrary orders.

Example 3. Calculate the multiple logic derivative for $f\left(x_{1}, x_{2}, x_{3}, x_{4}, x_{5}\right)=x_{1}\left(x_{2} \vee \bar{x}_{4}\right) \vee x_{2} \bar{x}_{3} x_{5} \vee$ $\vee \bar{x}_{2} \bar{x}_{3} x_{4} \bar{x}_{5}$ with respect to the variables $x_{4}$ and $x_{5}$ by numeric set-theoretical method (this $f$ is borrowed from [21, see exercise 4.13, p. 87], where it be calculated using the XBOOLE algorithm). 
Solution. The given function $f$ has STF $Y^{1}=\{(11--),(1--0-),(-10-1),(-0010)\}^{1}$ and its canonical STF is $Y^{1}=\{(00010),(01001),(01011),(10000),(10001),(10010),(10100),(10101),(11001),(11010),(11110)$, $(11111)\}^{1}$. Hence the desired derivative we obtain (8) as:

$$
\begin{gathered}
Y^{1} \stackrel{\partial^{2} / \partial x_{4} \partial x_{5}}{\Rightarrow}\left\{l_{1} l_{2} l_{3}--\right\}=\{(000--),(010--),(010--),(100--),(100--),(100--),(101--),(101--), \\
(110--),(110--),(111--),(111--)\}^{\oplus} .
\end{gathered}
$$

After removal in the PSTF $Y^{\oplus}$ the pairs of identical elements we obtain a 2-nd order multiple STderivative: $\partial^{2} Y^{\oplus} / \partial x_{4} \partial x_{5}=\{(000--),(100--)\}^{\oplus}=(-00--)$.

Answer. $\partial^{2} f / \partial x_{4} \partial x_{5}=\bar{x}_{2} \bar{x}_{3}$ corresponds to [21].

Example 4. Calculate the multiple logic derivative for $f(a, b, c, d, e)=a \bar{c} \vee a b d \vee b d e \vee \bar{b} \bar{e}$ with respect to the variables $b$ and $c$ by numeric set-theoretical method (this $f$ is borrowed from [4] p. 307).

Solution. The given function $f$ has STF $Y^{1}=\{(1-0--),(11-1-),(-1-11),(-0--0)\}^{1}$, conjuncterms that are not mutually orthogonal. By transforming it into a canonical STF $Y^{1}=\{(00000),(00010)$, (00100),(00110),(01011),(01111),(10000),(10001),(10010), (10011),(10100),(10110),(11000),(11001), (11010),(11011),(11110),(11111) $\}^{1}$, we obtain the desired derivative (8) as:

$$
\begin{gathered}
Y^{1} \stackrel{\partial^{2} / \partial b \partial c}{\Rightarrow}\left\{l_{1}--l_{4} l_{5}\right\}=\{(0--00),(0--10),(0--00),(0--10),(0--11),(0--11),(1--00),(1--01), \\
\quad(1--10),(1--11),(1--00),(1--10),(1--00),(1--01),(1--10),(1--11),(1--10),(1--11)\}^{\oplus} .
\end{gathered}
$$

After removal the pairs of identical elements from this set we obtain a 2-nd order multiple STderivative: $\quad \partial^{2} Y^{\oplus} / \partial b \partial c=\{(1--00),(1--11)\}^{\oplus} \equiv\{(1--00),(1--11)\}^{1}$.

Answer. $\partial^{2} f / \partial b \partial c=a \bar{d} \bar{e} \vee$ ade corresponds to [4].

\subsection{Obtaining the $\boldsymbol{k}$-th order vectorial ST-derivative}

The analogical approach is used for numeric set-theoretical obtaining of a vectorial logic derivative of the $k$-th order (3), vectorial ST-derivative of $k$-th order for function $f\left(x_{1}, x_{2}, \ldots, x_{n}\right)$ with respect to some $k$ variables, $k \in\{1,2, \ldots, n\}$. As by definition (3) from the example of obtaining of vectorial logic derivative of the 2-order for function $f\left(x_{1}, \ldots, x_{i}, \ldots, x_{j}, \ldots, x_{n}\right)$ with respect to the variables $x_{i}$ and $x_{j}$, and taking into account (4) and (6) as well as properties of the mod-2-sum operation we have:

$$
\begin{gathered}
\frac{\partial^{2} f}{\partial\left(x_{i}, x_{j}\right)}=\frac{\partial f}{\partial x_{i}} \oplus \frac{\partial f}{\partial x_{j}} \oplus \frac{\partial^{2} f}{\partial x_{i} \partial x_{j}}=\left(f\left(x_{1}, \ldots, x_{i}, \ldots, x_{j}, \ldots, x_{n}\right) \oplus f\left(x_{1}, \ldots, \bar{x}_{i}, \ldots, x_{j}, \ldots, x_{n}\right)\right) \oplus \\
\oplus\left(f\left(x_{1}, \ldots, x_{i}, \ldots, x_{j}, \ldots, x_{n}\right) \oplus f\left(x_{1}, \ldots, x_{i}, \ldots, \bar{x}_{j}, \ldots, x_{n}\right)\right) \oplus \\
\oplus\left(f\left(x_{1}, \ldots, x_{i}, \ldots, x_{j}, \ldots, x_{n}\right) \oplus f\left(x_{1}, \ldots, x_{i}, \ldots, \bar{x}_{j}, \ldots, x_{n}\right)\right) \oplus\left(f\left(x_{1}, \ldots, \bar{x}_{i}, \ldots, x_{j}, \ldots, x_{n}\right) \oplus f\left(x_{1}, \ldots, \bar{x}_{i}, \ldots, \bar{x}_{j}, \ldots, x_{n}\right)\right) .
\end{gathered}
$$

Hence, after removal of identical pairs of formed functions we get:

$$
\frac{\partial^{2} f}{\partial\left(x_{i}, x_{j}\right)}=f\left(x_{1}, \ldots, x_{i}, \ldots, x_{j}, \ldots, x_{n}\right) \oplus f\left(x_{1}, \ldots, \bar{x}_{i}, \ldots, \bar{x}_{j}, \ldots, x_{n}\right) .
$$

Is easier compared to (3) calculate the vectorial logic derivative by formula (9). For example, a vectorial derivative for minterm $\bar{x}_{1} \bar{x}_{2} \bar{x}_{3}$ with respect to $x_{1}$ and $x_{2}$ by the formula (3) is obtained this way:

$$
\frac{\partial^{2}}{\partial\left(x_{1}, x_{2}\right)}\left(\bar{x}_{1} \bar{x}_{2} \bar{x}_{3}\right)=\frac{\partial}{\partial x_{1}}\left(\bar{x}_{1} \bar{x}_{2} \bar{x}_{3}\right) \oplus \frac{\partial}{\partial x_{2}}\left(\bar{x}_{1} \bar{x}_{2} \bar{x}_{3}\right) \oplus \frac{\partial^{2}}{\partial x_{1} \partial x_{2}}\left(\bar{x}_{1} \bar{x}_{2} \bar{x}_{3}\right)=\bar{x}_{2} \bar{x}_{3} \oplus \bar{x}_{1} \bar{x}_{3} \oplus \bar{x}_{3}=
$$




$$
=\left(\bar{x}_{2} \oplus \bar{x}_{1} \oplus 1\right) \bar{x}_{3}=\left(\bar{x}_{1} \bar{x}_{2} \vee x_{1} x_{2}\right) \bar{x}_{3}=\bar{x}_{1} \bar{x}_{2} \bar{x}_{3} \vee x_{1} x_{2} \bar{x}_{3},
$$

and by the formula (9): $\frac{\partial^{2}}{\partial\left(x_{1}, x_{2}\right)}\left(\bar{x}_{1} \bar{x}_{2} \bar{x}_{3}\right)=\bar{x}_{1} \bar{x}_{2} \bar{x}_{3} \oplus x_{1} x_{2} \bar{x}_{3}$.

As in the previous cases, the obtained result of the vectorial logic differentiation, if needed, can be rewritten in the SOP $f$, with replacing the symbol $\oplus$ by the symbol $\vee$. In the above example we will have $\frac{\partial^{2}}{\partial\left(x_{1}, x_{2}\right)}\left(\bar{x}_{1} \bar{x}_{2} \bar{x}_{3}\right)=\bar{x}_{1} \bar{x}_{2} \bar{x}_{3} \vee x_{1} x_{2} \bar{x}_{3}$.

Respectively, the vectorial logic derivative of the $n$-th order for the function $f\left(x_{1}, x_{2}, \ldots, x_{n}\right)$ with respect to all variables $x_{1}, x_{2}, \ldots, x_{n}$ is:

$$
\frac{\partial^{n} f}{\partial\left(x_{1}, x_{2}, \ldots, x_{n}\right)}=f\left(x_{1}, x_{2}, \ldots, x_{n}\right) \oplus f\left(\bar{x}_{1}, \bar{x}_{2}, \ldots, \bar{x}_{n}\right)
$$

From the expression (9) it follows out that for obtaining the vectorial ST-derivative with respect to $x_{i}$ and $x_{j}$ (the operator $\stackrel{\partial^{2} / \partial\left(x_{i}, x_{j}\right)}{\Rightarrow}$ ), i.e. $\partial^{2} Y^{\oplus} / \partial\left(x_{i} x_{j}\right)$, it is necessary to apply two masks of literals: $\left\{l_{1} \cdots l_{i} \cdots l_{j} \cdots l_{n}\right\}$, using which the given minterms $m_{1}, m_{2}, \ldots, m_{p}$ are rewritten, and $\left\{l_{1} \cdots \bar{l}_{i} \cdots \bar{l}_{j} \cdots l_{n}\right\}$ by which the positions of binary minterms the corresponding variable $\left(x_{i}\right.$ and $\left.x_{j}\right)$ are inverted with respect to which the derivative is sought. Due to that, (for the sake of clarity, the masks are shown in the column $\left.\left\{\begin{array}{l}l_{1} \cdots l_{i} \cdots l_{j} \cdots l_{n} \\ l_{1} \cdots \bar{l}_{i} \cdots \bar{l}_{j} \cdots l_{n}\end{array}\right\}\right)$, we obtain the PSTF $Y^{\oplus}=\left\{\begin{array}{l}m_{1}, m_{2}, \ldots, m_{p}, \\ m_{1}^{*}, m_{2}^{*}, \ldots, m_{p}^{*}\end{array}\right\}$, where $m_{q}^{*}=\left(\sigma_{1} \cdots \bar{\sigma}_{i} \cdots \bar{\sigma}_{j} \cdots \sigma_{n}\right)$ is the $q$-th minterm formed by application of the mask $\left\{l_{1} \cdots \bar{l}_{i} \cdots \bar{l}_{j} \cdots l_{n}\right\}$ on the $q$-th minterm $m_{q}=\left(\sigma_{1} \cdots \sigma_{i} \cdots \sigma_{j} \cdots \sigma_{n}\right)$ of the canonical STF $Y^{1}=\left\{m_{1}, m_{2}, \ldots, m_{p}\right\}^{1}$ of the function $f$, i.e.:

$$
\begin{gathered}
Y^{1}=\left\{m_{1}, m_{2}, \ldots, m_{p}\right\}^{1} \stackrel{\partial^{2} / \partial\left(x_{i}, x_{j}\right)}{\Rightarrow}\left\{\begin{array}{c}
l_{1} \cdots l_{i} \cdots l_{j} \cdots l_{n} \\
l_{1} \cdots \bar{l}_{i} \cdots \bar{l}_{j} \cdots l_{n}
\end{array}\right\}=\left\{\begin{array}{l}
m_{1}, m_{2}, \ldots, m_{p}, \\
m_{1}^{*}, m_{2}^{*}, \ldots, m_{p}^{*}
\end{array}\right\} \Rightarrow\left\{m_{\lambda_{1}}, m_{\lambda_{2}}, \ldots, m_{\lambda_{p^{*}}}\right\}^{\oplus} \Rightarrow \\
\Rightarrow\left\{\theta_{1}^{r_{1}}, \theta_{2}^{r_{2}}, \ldots, \theta_{s}^{r_{s}}\right\},
\end{gathered}
$$

where $\left\{m_{\lambda_{1}}, m_{\lambda_{2}}, \ldots, m_{\lambda_{p^{*}}}\right\}^{\oplus}$ is the set of minterms obtained after removal of identical pairs of elements; $\left\{\theta_{1}^{r_{1}}, \theta_{2}^{r_{2}}, \ldots, \theta_{s}^{r_{s}}\right\}$ is the vectorial ST-derivative $\partial^{2} Y^{\oplus} / \partial\left(x_{i}, x_{j}\right)$ formed by minimization procedure; $s \leq p^{*}$.

As one can see, the definition of the $k$-th order vectorial ST-derivative differs from the considered algorithm for determining multiple ST-derivative of the $k$-th order (see Section 2.4) only in the way that on the given minterms of the function $f$ are overlay two masks with noninverted and inverted literals, where the last ones mask exactly those variables with respect to which the derivative is sought.

Example 5. To calculate the 2-nd order vectorial logic derivative for the function $f\left(x_{1}, x_{2}, x_{3}\right)=$ $=x_{1} x_{2} \vee x_{1} \bar{x}_{3}$ with respect to $x_{1}$ and $x_{2}$ by numeric set-theoretical method (this $f$ is borrowed from [19] example 2.5 , p. 65).

Solution. The given function $f$ has STF $Y^{1}=\{(11-),(1-0)\}^{1}$ and its canonical STF $Y^{1}=\{100,110,111\}^{1}$. By the formula (10) we have:

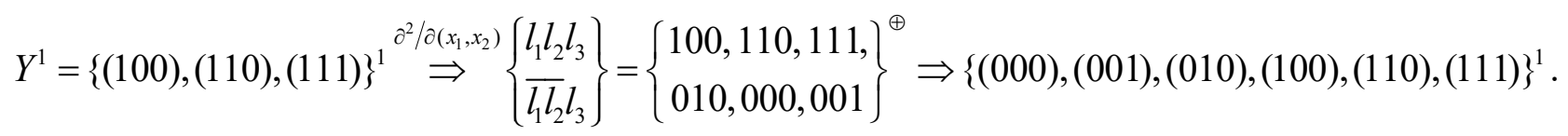


After minimization of the obtained canonical STF $Y^{1}$ the searched the 2-order vectorial ST-derivative $\partial^{2} Y^{\oplus} / \partial\left(x_{1}, x_{2}\right) \Rightarrow\{(--0),(00-),(11-)\}^{1}$.

Answer. $\partial^{2} f / \partial\left(x_{1}, x_{2}\right)=\bar{x}_{3} \vee \bar{x}_{1} \bar{x}_{2} \vee x_{1} x_{2}$ that corresponds to [19].

Example 6. To calculate the 1-st order derivative $\partial f / \partial x_{1}$ and the 2-nd order derivatives $\partial^{2} f / \partial x_{1} \partial x_{2}$ and $\partial^{2} f / \partial\left(x_{1}, x_{2}\right)$ for the function $f=x_{1} x_{2} \vee x_{1} x_{3} \vee x_{2} x_{3}$ by numeric set-theoretical method (this $f$ is borrowed from [26], example 6.2.1, p. 125).

Solution. The given function $f$ has STF $Y^{1}=\{(11-),(1-1),(-11)\}^{1}$ and its canonical STF is $Y^{1}=\{(011),(101),(110),(111)\}^{1}$.

The simple ST-derivative can be obtained as

$$
Y^{1}=\{(011),(101),(110),(111)\}^{1} \stackrel{\partial / \partial x_{1}}{\Rightarrow}\left\{-l_{2} l_{3}\right\}=\{(-11),(-01),(-10),(-11)\}^{\oplus} \Rightarrow\{(-01),(-10)\}^{\oplus} .
$$

Therefore, $\partial Y^{\oplus} / \partial x_{1}=\{(-01),(-10)\}^{\oplus} \equiv\{(-01),(-10)\}^{1}$.

Thus, the multiple ST-derivative can be obtained as

$$
Y^{1}=\{(011),(101),(110),(111)\}^{1} \stackrel{\partial / \partial x_{1} \partial x_{2}}{\Rightarrow}\left\{--l_{3}\right\}=\{(--1),(--1),(--0),(--1)\}^{\oplus}=\{(--0),(--1)\}^{\oplus} \Rightarrow\{(---)\}^{1} .
$$

Therefore, $\partial^{2} Y^{\oplus} / \partial x_{1} \partial x_{2}=\{(---)\}^{1} \equiv \mathbf{1}$.

The vectorial ST-derivative is

$$
Y^{1}=\{(011),(101),(110),(111)\}^{1} \stackrel{\partial^{2} / \partial\left(x_{1}, x_{2}\right)}{\Rightarrow}\left\{\begin{array}{l}
l_{1} l_{2} l_{3} \\
\bar{l}_{1} l_{2} l_{3}
\end{array}\right\}=\left\{\begin{array}{l}
011,101,110,111, \\
101,011,000,001
\end{array}\right\}^{\oplus} .
$$

Therefore, $\partial^{2} Y^{\oplus} / \partial\left(x_{1}, x_{2}\right)=\{(000),(001),(110),(111)\}^{\oplus} \equiv\{(00-),(11-)\}^{1}$.

Answer. $\partial f / \partial x_{1}=\bar{x}_{2} x_{3} \vee x_{2} \bar{x}_{3}=x_{2} \oplus x_{3} ; \partial^{2} f / \partial x_{1} \partial x_{2}=1 ; \partial^{2} f / \partial\left(x_{1}, x_{2}\right)=\bar{x}_{1} \bar{x}_{2} \vee x_{1} x_{2}=\overline{x_{1} \oplus x_{2}}=x_{1} \sim x_{2}$ that corresponds to [26].

Example 7. To calculate the 2-nd order vectorial derivative for $f(a, b, c, d, e)=a \bar{c} \vee a b d \vee b d e \vee \bar{b} \bar{e}$ with respect to the variables $a$ and $e$ by numeric set-theoretical method (this fis borrowed from [4], p. 309). Solution. The given function $f$ has STF $Y^{1}=\{(1-0--),(11-1-),(-1-11),(-0--0)\}^{1}$ and its canonical STF is $Y^{1}=\{(00000),(00010),(00100),(00110),(01011),(01111),(10000),(10001),(10010),(10011),(10100)$, (10110),(11000),(11001),(11010),(11011),(11110),(11111) $\}^{1}$.

$$
\begin{aligned}
Y^{1} \stackrel{\partial^{2} / \partial(a, e)}{\Rightarrow}\left\{\begin{array}{l}
l_{1} l_{2} l_{3} l_{4} l_{5} \\
\overline{l_{1}} l_{2} l_{3} l_{4} \bar{l}_{5}
\end{array}\right\}=\left\{\begin{array}{r}
00000,00010,00100,00110,01011,01111,10000,10001,10010,10011, \\
10100,10110,11000,11001,11010,11011,11110,11111, \\
10001,10011,10101,10111,11010,11110,00001,00000,00011,00010, \\
00101,00111,01001,01000,01011,01010,01111,01110
\end{array}\right\}^{\oplus} \Rightarrow \\
\Rightarrow\{(00001),(00011),(00100),(00101),(00110),(0011),(01000),(01001),(01010),(01110), \\
(10000),(10010),(10100),(10101),(10110),(10111),(11000),(11001),(11011),(11111)\}^{1} .
\end{aligned}
$$

After minimization of the obtained canonical STF $Y^{1}$ of the given function $f$ [20] the searched vectorial ST-derivative is $\partial^{2} Y^{\oplus} / \partial(a, e) \Rightarrow\{(-01--),(00--1),(-100-),(10--0),(01-10),(11-11)\}^{1}$.

Answer. $\partial^{2} f / \partial(a, e)=\bar{b} c \vee \bar{a} \bar{b} e \vee b \bar{c} \bar{d} \vee a \bar{b} \bar{e} \vee \bar{a} b d \bar{e} \vee a b d e$ that corresponds to [4].

Example 8. To calculate the 3-th order vectorial logic derivative for the function $f\left(x_{1}, x_{2}, x_{3}\right)$ that is given by canonical STF is $Y^{1}=\{(100),(110),(111)\}^{1}$, with respect to the variables $x_{1}, x_{2}$ and $x_{3}$ by numeric set-theoretical method (this $f$ is borrowed from [19], p. 66). 
Solution. According to formula (11) we have:

$$
\begin{gathered}
Y^{1}=\{(100),(110),(111)\}^{1^{3} / \partial\left(x_{1}, x_{2}, x_{3}\right)} \Rightarrow\left\{\begin{array}{l}
l_{1} l_{2} l_{3} \\
\bar{l}_{1} l_{2} l_{3}
\end{array}\right\}=\left\{\begin{array}{l}
100,110,111,{ }^{\oplus} \\
011,001,000
\end{array}\right\}^{\oplus} \Rightarrow \\
\Rightarrow\{(000),(001),(011),(100),(110),(111)\}^{1} .
\end{gathered}
$$

After minimization of the obtained set, the vectorial ST-derivative for the given function is $\partial^{3} Y^{\oplus} / \partial\left(x_{1}, x_{2}, x_{3}\right) \Rightarrow\{(-00),(0-1),(11-)\}^{1}$.

Answer. $\partial^{3} f / \partial\left(x_{1}, x_{2}, x_{3}\right)=\bar{x}_{2} \bar{x}_{3} \vee \bar{x}_{1} x_{3} \vee x_{1} x_{2}$ that corresponds to [19].

\section{On the effectiveness of the proposed method}

The basis of the effective assessment of the proposed numerical set-theoretic method for determining logic derivatives of arbitrary orders compared to known methods is the number of procedural steps necessary to achieve the goal.

As one can see in Sections 2.2-2.4, the algorithm of the proposed method envisages only two procedural steps (the minimization of the obtained result can be considered as the third one, which does not matter), and regardless of $n$ and $k$, namely: 1) overlaying a mask for binary literals of the $(n-k)$ - rank on binary minterms of the given function $f ; 2$ ) removing the pairs of identical ternary conjuncterms of the $(n-k)$-rank in the set PSTF $Y^{\oplus}$.

When calculating the $k$-th order vectorial ST-derivative (see Section 2.4) instead of the mask of the $(n-k)$ - rank we can apply simultaneously two masks of the $n$-rank, one of which has $k$ inverted literals. However, this does not affect the number of procedural steps.

The main drawback of the known methods of the logic derivatives calculation $[2-8,11-13,17,21$, $25]$ is that they are based on an analytical approach $[1,19,26]$, where the number of procedural steps heavily depends on the $n$ and $k$. An example that reflects analytical calculation of the logic derivatives using a computer is the software package XBOOLE [21]. We illustrate this drawback on a simple example of calculation of the 1-st order logic derivative $(k=1)$.

As stated in [4, p. 305], for differentiation of a function $f$ with respect to one variable we must first assign this variable values 1 and 0 consequently and execute the appropriate substitution in the formula of the function $f$. As a result, then perform the mod-2-sum operation on the obtained subfunctions, that do not depend on this variable, as well as rather complicated further conversions. So, if $k=1$, we must perform three procedural steps. For $k>1$ the assignment procedure will consist of more than three steps, which accordingly complicates further transformations. For example, if $k=2$, we need consequently assign four values 00,01 10,11 to the variables whose 2 -nd order mixed derivative is calculated and, respectively, we need $2^{k}$ for $k$ values. Using the proposed method for arbitrary $k$ and $n$ it is enough to overlay on the given binary minterms only the mask of literals of the $(n-k)$-rank that simplifies the further conversions.

Thus, the computational complexity of determining the different types of the $k$-th order logic derivatives by the proposed algorithm is relatively lower.

\section{Conclusions}

The described new numeric set-theoretical method for determination of the different logical derivatives types of the arbitrary orders differs by simplicity of realization if compared to the known methods. In particular, the definition of multiple ST-derivative of the $k$-th order does not require to perform stepby-step identification of appropriate derivatives of the 1-st order and previous assignment procedure. The proposed algorithm has relatively less computational complexity due to lower amount of the operations required for solving the given problem. This is proved in the given examples borrowed from wellknown publications, in order to compare them with the proposed numeric set-theoretic method to obtain the different logic derivatives types of the arbitrary order. 
1. Thayse A. Boolean Differential Calculus. - Germany, Heidelberg: Springer-Verlag, 1980. -367 p.

2. Steinbach B., Posthoff C. Boolean Differential Calculus / Sasao T., Butler J.T. Progress in Appl. of Boolean Functions. 2010. - P. 55-78.

3. Astola J.T., Stankovic R.S. Fundamentals of Switching Theory and Logic Design. - Springer, 2006. - P. 235-250.

4. Закревский А.Д., Поттосин Ю.В., Черемисинова Л.Д. Логические основы проектирования дискретных устройств. М.: Физматлит, 2007. - 592 с.

5. Logic Differential Calculus: Achievements, Trends and Applications / S. Yanushkevich, D. Bochmann, R. Stankovic et al. // J. Avtomatika and Telemechanika. - 2000. - N 6. - P. 155-170.

6. Levashenko V., Shmerko V., Yanushkevich S. Solution of Boolean Differential Equations on Systolic Arrays // Cybernetics and System Analysis. - 32, N 1. - P. 26-40 RUS: PDF FILE.

7. Logic Differential Calculus as Part of Switching Theory / D. Bochmann, Ch. Posthoff, V. Shmerko et al. // IEE 4-th Int. Conf. on New Inform. Technol. in Educ. - NITE'2000, Minsk, Belarus. - 2000. - P. 126-135 PostScript FILE.

8. Yanushkevich S.N. Matrix and Combinatorics Solution of Boolean Differential Equations // Discrete Applied Mathematics, Preprint submitted to Elsevier Preprint, 2001 PostScript FILE.

9. Sellers F.F., Hsiao M.Y., Bearson L.W. Analizing errors with the Boolean difference // IEEE Trans. Comp. - 1968. - N 1. P. 676-683.

10. Marinos $P$. Derivation of minimal complete sets of test-input sequences using Boolean differences // IEEE Trans. Computers. - 1981. - C-20, N 1. - P. 25-32.

11. Steinbach B., Zhang Z., Lang Ch. Logical design of fully testable large circuits by decomposition // Proc. Int. Conf. On Computers-Aided Design of Discrete Devices. Minsk. Belarus. - 1997. - P. 7-14.

12. Jaakko T.A., Stankovic R.S. Fundamentals of Switching Theory and Logic Design. A Hands on Approach. Springer. 2006. $-342 \mathrm{p}$.

13. Yanushkevich S. Matrix method to solve logic differential equations // Computers and Digital Technique (UK). - 1997. - 144, N 5. - P. 267-272.

14. Marincovic S., Tosic Z. Algorithm for minimal polarized form determination // IEEE Trans. Computers. - 1974. - C-23, N 12. - P. 1313-1315.

15. Davio M., Deschamps J. Symmetric discrete functions // Philips Res. Repts. - 1972. - 27. N 5. - P. 405-445.

16. Супрун В.П. Полиномиальное разложение симметрических булевых функций // Изв. АН СССР. Техн. кибернетика. - 1985. - № 5. - С. 123-127.

17. Yanushkevich S.N. Logic differential calculus in multivalued logic design // Tech. University of Szczecin Akad. Publ., Poland. - 1998. - P. 32-39.

18. Zaitseva E.N. Applications of Logic Differential Calculus in CAD of Integrated Circuits, PhD Thesis, Univ. of Informatics and Radioelectronics, Minsk, Belarus. - 1993. - P. 45-59.

19. Горбатов В.А. Основы дискретной математики. - М.: Высш. шк., 1986. - 311 с.

20. Рицар Б.С. Простий візуальний метод визначення логікових похідних довільних порядків // Відбір і обробка інформації. - 2013. - 38(114). - С. 125-132.

21. Steinbach B., Posthoff C. Logic Functions and Equations. Examples and Exercises. - Springer Science + Business Media B.V. $-2009 .-230 \mathrm{p}$.

22. Rytsar B., Romanowski P., Shvay A. Set-theoretical Constructions of Boolean Functions and theirs Appl. in Logic Synthesis // Fundamenta Informaticae. - 2010. - 99, N 3. - P. 339-354.

23. Рицар Б.С. Числова теоретико-множинна інтерпретація полінома Жегалкіна // УСиМ. - 2013. - № 1. - С. 11-26.

24. Рицар Б.С. Числова теоретико-множинна інтерпретація поліномів Ріда-Маллера з фіксованою та змішаною полярністю // Там же. - № 3. - С. 30-50.

25. Butler J.T., Sasao T. Boolean Functions for Cryptography / Sasao T., Butler J.T.: Progress in Appl. of Boolean Functions. $-2010 .-$ P. 33-53.

26. Schneeweiss W.G. Boolean Functions with Engineering Applications and Computer Programs. - Berlin Heidelberg: Springer-Verlag 1989. - $264 \mathrm{p}$.

Поступила 12.09.2016

E-mail: bohdanrytsar@gmail.com

(C) Б.Е. Рыцар, 2016 